\title{
Maximum Principle for Forward-Backward Control System Driven by Itô-Lévy Processes under Initial-Terminal Constraints
}

\author{
Meijuan Liu, Xiangrong Wang, and Hong Huang \\ College of Mathematics and Systems Science, Shandong University of Science and Technology, Qingdao 266590, China \\ Correspondence should be addressed to Xiangrong Wang; xrwang2000@126.com
}

Received 13 January 2017; Revised 14 April 2017; Accepted 4 May 2017; Published 20 June 2017

Academic Editor: Zhongwei Lin

Copyright (C) 2017 Meijuan Liu et al. This is an open access article distributed under the Creative Commons Attribution License, which permits unrestricted use, distribution, and reproduction in any medium, provided the original work is properly cited.

\begin{abstract}
This paper investigates a stochastic optimal control problem where the control system is driven by Itô-Lévy process. We prove the necessary condition about existence of optimal control for stochastic system by using traditional variational technique under the assumption that control domain is convex. We require that forward-backward stochastic differential equations (FBSDE) be fully coupled, and the control variable is allowed to enter both diffusion and jump coefficient. Moreover, we also require that the initial-terminal state be constrained. Finally, as an application to finance, we show an example of recursive consumption utility optimization problem to illustrate the practicability of our result.
\end{abstract}

\section{Introduction}

The stochastic optimal control problem is very important in control theory. The sufficient and necessary conditions for the optimal control, called the maximum principle, are one of the control topics. Since 1960s, Pontryagin gave the necessary maximum principle under the deterministic control system; a lot of work has been done on this topic. Especially in nearly 20 years, many important results have been obtained; see literatures [1-4]. One can refer to Yong and Zhou [5], Wu [6], or Peng and Wu [7], for a complete account on the subject of maximum principle and a complete list of references.

In recent years, motivated by studying the mathematical economics and mathematical finance, many scholars turned their sight on forward-backward stochastic control system (FBSCS in short). Initially, they studied the optimal conditions about the control system driven by Brownian motion and obtained some fundamental results in many literatures. Ji and Zhou [8] studied a kind of forward-backward stochastic control system where the forward state is constrained in a convex set at the terminal time, and a stochastic maximum principle is obtained. Liu et al. [9] considered one kind of linear quadratic optimal control with constraint for discretetime stochastic systems with state and disturbance dependent noise, presenting a necessary condition under which the problem is well posed and a state feedback solution can be derived. $\mathrm{Wu}$ and $\mathrm{Xu}[10]$ obtained the maximum principle for fully coupled FBSCS with state constraints, in which the control domain needs to be convex and the diffusion coefficient does not contain control variables. Based on the results of $\mathrm{Wu}$ and Xu's work in [10], Shi [11] studied a fully coupled forward-backward control system where the forward diffusion coefficient does not contain the control variable, but control domain is not necessarily convex. For the above-mentioned question, Meng [12] and Ji and Wei [13] have applied the terminal variation approach to obtain the maximum principle under other versions.

Considering the complexity of finance market, as a consequence, it becomes natural to investigate control problems for systems driven by one kind of Itô-Lévy processes which is introduced by Nualart and Shoutens [14] and detailedly described by Applebaum [15]. Many researches have been done to extend the stochastic maximum principle of stochastic differential equations (SDEs in short) involving some Lévy jumps. Motivated by risk minimization, Øksendal and Sulem [16] studied the partial coupled FBSCS which need control domain to be convex and obtained maximum principle. Shi and Wu [17] obtained both necessary and sufficient maximum principle for optimal control of stochastic system with random jumps consisting of forward-backward state 
variables. The control variable is allowed to enter both diffusion and jump coefficients. Based on this literature, Shi [18] proved the global result of maximum principle where the control domain is not assumed to be convex, and the control variable appears in both diffusion and jump coefficient of the forward equation. Zhang et al. [19] discussed the stochastic optimal control question of FBSDE driven by Teugels martingales and obtained the maximum principle under the assumption of convex control domain.

In many classical cases as well as recent studies of forward-backward stochastic systems, introducing the model to formulate the process of financial derivative products has been a valid method to solve questions. As application of stochastic control theory, the stochastic maximum principle is usually used to solve investment strategy, risk control, and recursive utility; see examples of the literature: Ji and Zhou [8], Nualart and Schoutens [14], Shi and Wu [17], and Aase [20]. Similarly, we also apply the maximum principle to study the problem of maximizing consumption utility with initialterminal state restrained, which is also discussed by $\emptyset \mathrm{ksendal}$ and Sulem [21].

In this paper, we extend the result of Shi and $\mathrm{Wu}$ [17] to the case of fully coupled FBSCS, in which the control system is driven by Itô-Lévy process. At the same time, we demand that this forward-backward control system be constrained about initial-terminal state. We firstly use the traditional convex variational technique to prove the necessary condition about optimality. Then, using the result of this paper, we consider the problem of recursive consumption utility optimization problem with initial-terminal state constraints.

This paper is organized as follows: In Section 2, we formulate the control problem and list some preliminaries for fully coupled FBSCS. In Section 3, we establish variational equations about one kind of FBSCS with Itô-Lévy jumps in general form and then obtain variational inequality. In Section 4, we obtain the necessary condition of optimal control problem. In Section 5, we apply our result to test the application about the recursive utility problems.

\section{Preliminaries and Notations}

Let $\left(\Omega, \mathscr{F}, \mathscr{F}_{t}, P ; t \geq 0\right)$ be a complete filtration space and $\left\{\mathscr{F}_{t} ; t \geq 0\right\}$ be a filtration satisfying the usual conditions. On the above filtration space there exist two mutually independent stochastic processes:

(1) A d-dimensional Brownian motion $\left\{B_{t} ; t \geq 0\right\}$

(2) A Poisson random measure $N$ on $R^{+} \times E$, where $E=$ $R-\{0\}$ with the Borel $\sigma$-field $\mathscr{B}(E): \lambda$ is the intensity of $N$ with the property that $\int_{E}\left(1 \wedge|e|^{2}\right) \lambda(d e)<\propto$ and $\bar{N}$ is the compensator of $N, \bar{N}(d e, d t)=\lambda(d e) d t$; then $\{\widetilde{N}\}((0, t] \times E)=(N-\bar{N})((0, t] \times E)_{t \geq 0}$ is a martingale for all $\mathscr{A} \in \mathscr{B}(E)$ satisfying $\lambda(\mathscr{A})<\propto$.

Also we introduce the following notations:

$\tau$ : it is the transpose of a matrix.

$\langle\alpha, \beta\rangle$ : it is the inner product in $R^{n}, \forall \alpha, \beta \in R^{n}$. $|\alpha|=\sqrt{\langle\alpha, \alpha\rangle}$ : it is the norm in $R^{n}, \forall \alpha \in R^{n}$.

$\langle A, B\rangle=\operatorname{tr}\left(A B^{\tau}\right)$ : it is the inner product in $R^{n \times m}$, $\forall A, B \in R^{n \times m}$.

$|A|=\sqrt{\operatorname{tr}\left(A A^{\tau}\right)}$ : it is the inner product in $R^{n \times m}, \forall A \in$ $R^{n \times m}$.

$\mathscr{L}^{2}\left(\Omega, \mathscr{F}_{T}, P\right):=\left\{\xi \mid \xi\right.$ is $\mathscr{F}_{T}$-measurable, $E|\xi|^{2}<$ $\propto\}$.

$\mathscr{M}^{2}(0, T):=\left\{\varphi(t) \mid \varphi(t)\right.$ is $\mathscr{F}_{t}$-predictable, $E \int_{0}^{T}|\varphi(t)|^{2}<$ $\propto\}$.

$F_{N}^{2}(0, T):=\left\{\varphi(t) \mid \varphi(t)\right.$ is $\mathscr{F}_{t}$-predictable, $E \int_{0}^{T} \int_{E} \mid \varphi(t$, $\left.e)\left.\right|^{2} \pi(d e) d t<\propto\right\}$.

Consider the following fully coupled forward-backward stochastic differential equation:

$$
\begin{aligned}
d x(t) & \\
= & b(t, x(t), y(t), z(t), k(\mathrm{t}, e)) d t \\
& +\sigma(t, x(t), y(t), z(t), k(t, e)) d B_{t} \\
& +\int_{E} g(t, x(t), y(t), z(t), k(t, e)) \widetilde{N}(d e, d t), \\
-d y(t) & \\
= & f(t, x(t), y(t), z(t), k(t, e)) d t-z(t) d B_{t} \\
& -\int_{E} k(t, e) \widetilde{N}(d e, d t), \\
& \\
x_{0}= & a, \\
y_{T}= & h(x(T)),
\end{aligned}
$$

where $(x(t), y(t), z(t), k(t, e))$ take value in $R^{n} \times R^{m} \times R^{m \times d} \times$ $R^{m}$, and

$$
\begin{array}{r}
b: \Omega \times[0, T] \times R^{n} \times R^{m} \times R^{m \times d} \times R^{m} \longrightarrow R^{n}, \\
\sigma: \Omega \times[0, T] \times R^{n} \times R^{m} \times R^{m \times d} \times R^{m} \longrightarrow R^{n \times d}, \\
g: \Omega \times[0, T] \times R^{n} \times R^{m} \times R^{m \times d} \times R^{m} \longrightarrow R^{n}, \\
f: \Omega \times[0, T] \times R^{n} \times R^{m} \times R^{m \times d} \times R^{m} \longrightarrow R^{m}, \\
h: R^{n} \longrightarrow R^{m}, \\
k: \Omega \times[0, T] \times E \longrightarrow R^{m} .
\end{array}
$$

We use the notations $\mu=(x(t), y(t), z(t), k(t, e))^{\tau}, A(t, \mu)=$ $\left(-G^{\tau} f(t, \mu), G b(t, \mu), G \sigma(t, \mu), G g(t, \mu)\right)^{\tau}$, where $G$ is a given $n \times m$ full-rank matrix. Make the following assumptions: 
$\left(H_{1}\right)$ :

(i) $b, \sigma, g$ and $f$ is uniformly Lipschitz with respect to $\mu$,

(ii) $h(x)$ is uniformly Lipschitz with respect to $x \in R^{n}$,

(iii) for each $x, h(x)$ is in $\mathscr{L}^{2}\left(\Omega \cdot \mathscr{F}_{T} \cdot P\right)$,

(iv) $L(\omega, t, 0,0,0,0) \in \mathscr{M}^{2}(0, T), L=b, \sigma, f, g$ respectively, and $g(\omega, t, 0,0,0,0) \in F_{N}^{2}(0, T)$ for $(\omega, t) \in \Omega \times[0, T]$,

$$
\left(H_{2}\right):\left\{\begin{array}{l}
\left(\text { i) }\langle A(t, \mu)-A(t, \bar{\mu}), \mu-\bar{\mu}\rangle+\int_{E}\left\langle G^{\tau} \widehat{g}, \widehat{k}\right\rangle \lambda(d e) \leq-v_{1}|G \widehat{x}|^{2}-v_{2}\left(\left|G^{\tau} \hat{y}\right|^{2}+\left|G^{\tau} \widehat{z}\right|^{2}+\int_{E}\left|G^{\tau} \widehat{k}\right|^{2} \lambda(d e)\right),\right. \\
\text { (ii) }\langle h(x)-h(\bar{x}), G(x-\bar{x})\rangle \geq v_{3}|G \widehat{x}|^{2}, \\
\forall \widehat{\mu}=(\mu-\bar{\mu})=(\widehat{x}, \hat{y}, \widehat{z})=(x-\bar{x}, y-\bar{y}, z-\bar{z}), \hat{g}=g(t, \mu, k)-g(t, \bar{\mu}, \bar{k}),
\end{array}\right.
$$

where $v_{1}, v_{2}$, and $v_{3}$ are given nonnegative constants with $v_{1}+$ $v_{2}>0, v_{2}+v_{3}>0$.

Lemma 1 (see [22]). Assume $\left(H_{1}\right)$ and $\left(H_{2}\right)$ hold; then there is a unique adapted solution $(x(t), y(t), z(t), k(t, e)) \in$ $\mathscr{M}^{2}\left(0, T ; R^{n+m+m \times d}\right) \times F_{N}^{2}\left(0, T ; R^{m}\right)$, and $0 \leq t \leq T$ satisfied (1).

Now we consider the following fully coupled FBSDE controlled system:

$$
\begin{aligned}
& d x(t)=b(t, x(t), y(t), z(t), k(t, e), u(t)) d t \\
& \quad+\sigma(t, x(t), y(t), z(t), k(t, e), u(t)) d B_{t} \\
& \quad+\int_{E} g(t, x(t), y(t), z(t), k(t, e), u(t)) \widetilde{N}(d e, d t), \\
& -d y(t)=f(t, x(t), y(t), z(t), k(t, e), u(t)) d t \\
& \quad-z(t) d B_{t}-\int_{E} k(t, e) \widetilde{N}(d e, d t),
\end{aligned}
$$$$
0 \leq t \leq T
$$

$x_{0}=a$,

$y_{T}=h(x(T))$,

where $(x(t), y(t), z(t), k(t, e)) \in \mathscr{M}^{2}\left(0, T ; R^{n+m+m \times d}\right) \times F_{N}^{2}(0$, $\left.T ; R^{m}\right), u(t) \in U$, and $U \subset R^{k}$ is nonempty convex set, and mappings are

$$
\begin{aligned}
b: & \Omega[0, T] \times R^{n} \times R^{m} \times R^{m \times d} \times R^{m} \times \mathscr{U}_{\mathrm{ad}} \longrightarrow R^{n}, \\
\sigma: & \Omega \times[0, T] \times R^{n} \times R^{m} \times R^{m \times d} \times R^{m} \times \mathcal{U}_{\mathrm{ad}} \\
& \longrightarrow R^{n \times d}, \\
g: & \Omega \times[0, T] \times R^{n} \times R^{m} \times R^{m \times d} \times R^{m} \times \mathcal{U}_{\mathrm{ad}} \longrightarrow R^{n}, \\
f: & \Omega \times[0, T] \times R^{n} \times R^{m} \times R^{m \times d} \times R^{m} \times \mathscr{U}_{\mathrm{ad}} \longrightarrow R^{m} .
\end{aligned}
$$

Let $\mathcal{U}_{a d}=\left\{v(\cdot) \in \mathscr{M}^{2}\left(0, T ; R^{k}\right) ; v(t) \in U, 0 \leq t \leq T\right.$. a.e, a.s $\}$; an element of $\mathcal{U}_{a d}$ is called an admissible control.

We define the following cost function:

$$
\begin{aligned}
& J(v(\cdot)) \\
& \quad=E\left[\int_{0}^{T} l(t, x(t), y(t), z(t), k(t, \cdot), v(t)) d t\right. \\
& \quad+\Phi(x(T))+\gamma(y(0))],
\end{aligned}
$$

and the state variables of initial and terminal values satisfy the following constraint conditions:

$$
\begin{aligned}
& E\left[G_{1 x}(x(T))\right]=0, \\
& E\left[G_{0 y}(y(0))\right]=0,
\end{aligned}
$$

where

$l: \Omega \times[0, T] \times R^{n} \times R^{m} \times R^{m \times d} \longrightarrow R$,

$$
\begin{gathered}
\Phi: R^{n} \longrightarrow R, \\
G_{1}: R^{n} \longrightarrow R^{n_{1}},
\end{gathered}
$$

$$
n_{1} \leq n,
$$

$$
\begin{aligned}
& \gamma: R^{m} \longrightarrow R, \\
& G_{0}: R^{m} \longrightarrow R^{m_{1}}, \\
& \quad m_{1} \leq m .
\end{aligned}
$$

Problem 2. Find an admissible control $u(\cdot) \in \mathscr{U}_{\mathrm{ad}}$, such that

$$
J(u(\cdot))=\inf _{v(\cdot) \in \mathscr{U}_{\mathrm{ad}}} J(v(\cdot)) .
$$

We also assume that 


$$
\left(H_{3}\right):\left\{\begin{array}{l}
\text { (i) } b, \sigma, g, f, h, l, \Phi, \gamma, G_{1} \text { and } G_{0} \text { are continuous differentiable in their arguments, } \\
\text { (ii) the derivatives of } b, \sigma, g, f, h, l, G_{1} \text { and } G_{0} \text { are uniformly bounded, } \\
\text { (iii) } l_{x}, l_{y}, l_{z}, l_{k}, l_{v} \text { are bounded by } c(1+|x|+|y|+|z|+|k(\cdot)|+|v|), \\
\text { (iv) }|\gamma| \leq k_{2}\left(1+|y|^{2}\right),|\Phi| \leq k_{2}\left(1+|x|^{2}\right),\left|\Phi_{x}\right| \leq k_{2}(1+|x|),\left|\gamma_{y}\right| \leq k_{2}\left(1+|y|^{2}\right), k_{2}>0 .
\end{array}\right.
$$

Remark 3. According to the conclusion of Lemma 1, for the given admissible controller $v(\cdot)$, (6) has a unique adapted solution $(x(\cdot), y(\cdot), z(\cdot), k(\cdot, \cdot), v(\cdot))$.

\section{Variational Equations and Variational Inequality}

Now we recall Ekeland's variational principle.

Lemma 4 (Ekeland's variational principle; see [23]). Let $(V, d(\cdot, \cdot))$ be a complete metric space and $F(\cdot): V \rightarrow R$ be a proper lower semicontinuous function bounded from below, if for every $\varepsilon>0$, there exist $u \in V$, such that $F(u) \leq$ $\inf _{v \in V} F(v)+\varepsilon$; then there exists $u^{\varepsilon} \in V$, such that

$$
\begin{aligned}
& \text { (i) } F\left(u^{\varepsilon}\right) \leq F(u), \\
& \text { (ii) } d\left(u, u^{\varepsilon}\right) \leq \varepsilon, \\
& \text { (iii) } F(v)+\sqrt{\varepsilon} d\left(v, u^{\varepsilon}\right) \geq F\left(u^{\varepsilon}\right), \quad \forall v \in V .
\end{aligned}
$$

Define the following distance on $V$, the space of admissible control; then $(V, d(\cdot, \cdot))$ is a complete metric space:

$$
d(v(\cdot), u(\cdot))=E\left[\int_{0}^{T}|v(t)-u(t)|^{2} d t\right]^{1 / 2}
$$

Let $(x(\cdot), y(\cdot), z(\cdot), k(\cdot, \cdot), u(\cdot))$ be the optimal solution of problem, for $v(\cdot) \in \mathcal{U}_{\text {ad }}$; define

$$
\begin{aligned}
& F_{\varepsilon}(v(\cdot))=\left\{E\left|G_{1}\left(x^{v}(T)\right)\right|^{2}+E\left|G_{0}\left(y^{v}(0)\right)\right|^{2}\right. \\
& \left.+E|J(v(\cdot))-J(u(\cdot))+\varepsilon|^{2}\right\}^{1 / 2},
\end{aligned}
$$

where $\left(x^{v}(\cdot), y^{v}(\cdot), z^{v}(\cdot), k^{v}(\cdot)\right)$ is the trajectory of (6) corresponding to $v(\cdot)$. And according to Ekeland's variational principle, it is easy to get the following proposition.

\section{Proposition 5. If}

(i) $F_{\varepsilon}(v(\cdot)): \mathscr{U}_{a d} \longrightarrow R$ is continuous function,

(ii) $F_{\varepsilon}(v(\cdot))>0, \quad \forall v(\cdot) \in \mathcal{U}_{a d}$,

(iii) $F_{\varepsilon}(u(\cdot)) \leq \inf _{v(\cdot) \in \mathscr{U}_{a d}} F_{\varepsilon}(v(\cdot))+\varepsilon$,

then there exists $u^{\varepsilon}(\cdot) \in \mathcal{U}_{a d}$, such that

(i) $F_{\varepsilon}\left(u^{\varepsilon}(\cdot)\right) \leq F_{\varepsilon}(u(\cdot))=\varepsilon$,

(ii) $d\left(u(\cdot), u^{\varepsilon}(\cdot)\right) \leq \sqrt{\varepsilon}$,

(iii) $F_{\varepsilon}(v(\cdot))+\sqrt{\varepsilon} d\left(v(\cdot), u^{\varepsilon}(\cdot)\right) \geq F_{\varepsilon}\left(u^{\varepsilon}(\cdot)\right), \quad \forall v \in \mathscr{U}_{a d}$. $\forall \bar{u}(\cdot) \in \mathcal{U}_{\text {ad }}$, note $\widehat{u}(\cdot)=\bar{u}(\cdot)-u(\cdot), \widehat{u}^{\varepsilon}(\cdot)=\bar{u}(\cdot)-u^{\varepsilon}(\cdot), u_{\delta}^{\varepsilon}(\cdot)=$ $u^{\varepsilon}(\cdot)+\delta\left(\bar{u}(\cdot)-u^{\varepsilon}(\cdot)\right)$, due to the convexity of $\mathcal{u}_{\text {ad }}$, for $0 \leq$ $\delta \leq 1, u_{\delta}^{\varepsilon}(\cdot) \in \mathcal{U}_{a d}$.

Let $\left(x_{\delta}^{\varepsilon}(\cdot), y_{\delta}^{\varepsilon}(\cdot), z_{\delta}^{\varepsilon}(\cdot), k_{\delta}^{\varepsilon}(\cdot)\right)$ and $\left(x^{\varepsilon}(\cdot), y^{\varepsilon}(\cdot), z^{\varepsilon}(\cdot), k^{\varepsilon}(\cdot)\right)$ be the trajectory of (6) corresponding to $u_{\delta}^{\varepsilon}(\cdot)$ and $u^{\varepsilon}(\cdot)$, respectively; then $F_{\varepsilon}\left(u_{\delta}^{\varepsilon}(\cdot)\right)-F_{\varepsilon}\left(u^{\varepsilon}(\cdot)+\sqrt{\varepsilon} d\left(u_{\delta}^{\varepsilon}(\cdot), u^{\varepsilon}(\cdot)\right) \geq 0\right.$.

Consider the following variational equation:

$$
\begin{aligned}
& d x_{\delta}^{\prime}(t)=\left[b_{x}(t) x_{\delta}^{\prime}(t)+b_{y}(t) y_{\delta}^{\prime}(t)+b_{z}(t) z_{\delta}^{\prime}(t)\right. \\
& \left.+b_{k}(t) k_{\delta}^{\prime}(t, e)+b_{v}(t) \widehat{u}^{\varepsilon}(t)\right] d t+\left[\sigma_{x}(t) x_{\delta}^{\prime}(t)\right. \\
& +\sigma_{y}(t) y_{\delta}^{\prime}(t)+\sigma_{z}(t) z_{\delta}^{\prime}(t)+\sigma_{k}(t) k_{\delta}^{\prime}(t, e) \\
& \left.+\sigma_{v}(t) \widehat{u}^{\varepsilon}(t)\right] d B_{t}+\int_{E}\left[g_{x}(t) x_{\delta}^{\prime}(t)\right. \\
& \quad+g_{y}(t) y_{\delta}^{\prime}(t)+g_{z}(t) z_{\delta}^{\prime}(t)+g_{k}(t) k_{\delta}^{\prime}(t, e) \\
& \left.\quad+g_{v}(t) \widehat{u}^{\varepsilon}(t)\right] \widetilde{N}(d e, d t), \\
& -d y_{\delta}^{\prime}(t)=\left[f_{x}(t) x_{\delta}^{\prime}(t)+f_{y}(t) y_{\delta}^{\prime}(t)+f_{z}(t) z_{\delta}^{\prime}(t)\right. \\
& \left.+f_{k}(t) k_{\delta}^{\prime}(t, e)+f_{v}(t) \widehat{u}^{\varepsilon}(t)\right] d t-z_{\delta}^{\prime}(t) d B_{t} \\
& \quad-\int_{E} k_{\delta}^{\prime}(t, e) \widetilde{N}(d e, d t), \\
& x_{\delta}^{\prime}(0)=0, \\
& y_{\delta}^{\prime}=h_{x}\left(x_{\delta}(T)\right) x_{\delta}^{\prime},
\end{aligned}
$$

and according to assumption $\left(\mathrm{H}_{3}\right)$, one can verify (20) satisfying assumptions $\left(H_{1}\right)$ and $\left(H_{2}\right)$. Then it is easy to get, for a given $\widehat{u}^{\varepsilon}$, the fact that there exists a unique adapted solution $\left(x_{\delta}^{\prime}(\cdot), y_{\delta}^{\prime}(\cdot), z_{\delta}^{\prime}(\cdot), k_{\delta}^{\prime}(\cdot, \cdot)\right), 0 \leq t \leq T$ satisfying $(20)$.

Proposition 6. Assume $\left(\mathrm{H}_{3}\right)$ holds; then

$$
\begin{aligned}
\lim _{\delta \rightarrow 0} \frac{x_{\delta}^{\varepsilon}(t)-x^{\varepsilon}(t)}{\delta} & =x_{\delta}^{\prime}(t), \\
\lim _{\delta \rightarrow 0} \frac{y_{\delta}^{\varepsilon}(t)-y^{\varepsilon}(t)}{\delta} & =y_{\delta}^{\prime}(t), \\
\lim _{\delta \rightarrow 0} \frac{z_{\delta}^{\varepsilon}(t)-z^{\varepsilon}(t)}{\delta} & =z_{\delta}^{\prime}(t), \\
\lim _{\delta \rightarrow 0} \frac{k_{\delta}^{\varepsilon}(t, \cdot)-k^{\varepsilon}(t, \cdot)}{\delta} & =k_{\delta}^{\prime}(t, \cdot),
\end{aligned}
$$

where the limit is in $\mathscr{M}^{2}\left(0, T ; R^{n+m+m \times d}\right) \times F_{N}^{2}\left(0, T ; R^{m}\right)$. 
Proof. Let

$$
\begin{aligned}
& \widehat{x}^{\varepsilon}(t)=x_{\delta}^{\varepsilon}(t)-x^{\varepsilon}(t), \\
& \widehat{y}^{\varepsilon}(t)=y_{\delta}^{\varepsilon}(t)-y^{\varepsilon}(t), \\
& \widehat{z}^{\varepsilon}(t)=z_{\delta}^{\varepsilon}(t)-z^{\varepsilon}(t), \\
& \widehat{k}^{\varepsilon}(t)=k_{\delta}^{\varepsilon}(t, \cdot)-k^{\varepsilon}(t, \cdot),
\end{aligned}
$$

and the notations $\bigwedge_{\delta}^{\varepsilon}$ and $\bigwedge^{\varepsilon}$ denote $\left(x_{\delta}^{\varepsilon}(t), y_{\delta}^{\varepsilon}(t), z_{\delta}^{\varepsilon}(t)\right.$, $\left.k_{\delta}^{\varepsilon}(t, \cdot)\right)$ and $\left(x^{\varepsilon}(t), y^{\varepsilon}(t), z^{\varepsilon}(t), k^{\varepsilon}(t, \cdot)\right)$, respectively; then the equation (for simplicity, we omit the time sign $t$ )

$$
\begin{aligned}
& d \widehat{x}^{\varepsilon}(t)=\left[b\left(t, x_{\delta}^{\varepsilon}, y_{\delta}^{\varepsilon}, z_{\delta}^{\varepsilon}, k_{\delta}^{\varepsilon}(\cdot), u^{\varepsilon}+\delta \widehat{u}^{\varepsilon}\right)\right. \\
& \left.-b\left(t, x^{\varepsilon}, y^{\varepsilon}, z^{\varepsilon}, k^{\varepsilon}(\cdot), u^{\varepsilon}\right)\right] d t \\
& +\left[\sigma\left(t, x_{\delta}^{\varepsilon}, y_{\delta}^{\varepsilon}, z_{\delta}^{\varepsilon}, k_{\delta}^{\varepsilon}(\cdot), u^{\varepsilon}+\delta \widehat{u}^{\varepsilon}\right)\right. \\
& \left.-\sigma\left(t, x^{\varepsilon}, y^{\varepsilon}, z^{\varepsilon}, k^{\varepsilon}(\cdot), u^{\varepsilon}\right)\right] d B_{t} \\
& +\int_{E}\left[g\left(t, x_{\delta}^{\varepsilon}, y_{\delta}^{\varepsilon}, z_{\delta}^{\varepsilon}, k_{\delta}^{\varepsilon}(\cdot), u^{\varepsilon}+\delta \widehat{u}^{\varepsilon}\right)\right. \\
& \left.-g\left(t, x^{\varepsilon}, y^{\varepsilon}, z^{\varepsilon}, k^{\varepsilon}(\cdot), u^{\varepsilon}\right)\right] \widetilde{N}(d e, d t) \\
& -d \widehat{y}^{\varepsilon}(t)=\left[f\left(t, x_{\delta}^{\varepsilon}, y_{\delta}^{\varepsilon}, z_{\delta}^{\varepsilon}, k_{\delta}^{\varepsilon}(\cdot), u^{\varepsilon}+\delta \widehat{u}^{\varepsilon}\right)\right. \\
& \left.-f\left(t, x^{\varepsilon}, y^{\varepsilon}, z^{\varepsilon}, k^{\varepsilon}(\cdot), u^{\varepsilon}\right)\right] d t-\widehat{z}^{\varepsilon} d B_{t} \\
& \quad-\int_{E} \widehat{k}_{\delta}^{\varepsilon}(\cdot) \widetilde{N}(d e, d t), \\
& \widehat{x}^{\varepsilon}(0)=0, \\
& \widehat{y}^{\varepsilon}(T)=0,
\end{aligned}
$$

can be rewritten into the following simple form:

$$
\begin{aligned}
& d \widehat{x}^{\varepsilon}(t)=\left[b\left(t, \bigwedge_{\delta}^{\varepsilon}, u^{\varepsilon}+\delta \widehat{u}^{\varepsilon}\right)-b\left(t, \bigwedge^{\varepsilon}, u^{\varepsilon}\right)\right] d t \\
&+ {\left[\sigma\left(t, \bigwedge_{\delta}^{\varepsilon}, u^{\varepsilon}+\delta \widehat{u}^{\varepsilon}\right)-\sigma\left(t, \bigwedge^{\varepsilon}, u^{\varepsilon}\right)\right] d B_{t} } \\
&+ \int_{E}\left[g\left(t, \bigwedge_{\delta}^{\varepsilon}, u^{\varepsilon}+\delta \widehat{u}^{\varepsilon}\right)-g\left(t, \bigwedge^{\varepsilon}, u^{\varepsilon}\right)\right]
\end{aligned}
$$

$\cdot \widetilde{N}(d e, d t)$

$$
\begin{aligned}
& -d \widehat{y}^{\varepsilon}(t)=\left[f\left(t, \bigwedge_{\delta}^{\varepsilon}, u^{\varepsilon}+\delta \widehat{u}^{\varepsilon}\right)\right. \\
& \left.-f\left(t, \bigwedge^{\varepsilon}, u^{\varepsilon}\right)\right] d t-\widehat{z}^{\varepsilon} d B_{t}-\int_{E} \widehat{k}_{\delta}^{\varepsilon}(e)
\end{aligned}
$$

$\cdot \widetilde{N}(d e, d t)$

$\widehat{x}^{\varepsilon}(0)=0$,

$\widehat{y}^{\varepsilon}(T)=0$.
Sign

$$
\begin{aligned}
& \widehat{g}=g\left(t, \bigwedge_{\delta}^{\varepsilon}, u^{\varepsilon}\right)-g\left(t, \bigwedge^{\varepsilon}, u^{\varepsilon}\right), \\
& \widehat{g}_{\delta}=g\left(t, \bigwedge_{\delta}^{\varepsilon}, u^{\varepsilon}+\delta \widehat{u}^{\varepsilon}\right)-g\left(t, \bigwedge_{\delta}^{\varepsilon}, u^{\varepsilon}\right), \\
& \widehat{\sigma}_{\delta}=\sigma\left(t, \bigwedge_{\delta}^{\varepsilon}, u^{\varepsilon}+\delta \widehat{u}^{\varepsilon}\right)-\sigma\left(t, \bigwedge_{\delta}^{\varepsilon}, u^{\varepsilon}\right), \\
& \widehat{b}_{\delta}=b\left(t, \bigwedge_{\delta}^{\varepsilon}, u^{\varepsilon}+\delta \widehat{u}^{\varepsilon}\right)-b\left(t, \bigwedge_{\delta}^{\varepsilon}, u^{\varepsilon}\right), \\
& \widehat{f}_{\delta}=f\left(t, \bigwedge_{\delta}^{\varepsilon}, u^{\varepsilon}+\delta \widehat{u}^{\varepsilon}\right)-f\left(t, \bigwedge_{\delta}^{\varepsilon}, u^{\varepsilon}\right) .
\end{aligned}
$$

By applying Itô formula to $\left|\widehat{x}^{\varepsilon}\right|^{2},\left|\widehat{y}^{\varepsilon}\right|^{2}$, and $\left\langle G \widehat{x}^{\varepsilon}, \widehat{y}^{\varepsilon}\right\rangle$, we can prove that $\left(\widehat{x}^{\varepsilon}, \widehat{y}^{\varepsilon}, \widehat{z}^{\varepsilon}, \widehat{k}^{\varepsilon}\right)$ converges to 0 in $\mathscr{M}^{2}(0$, $\left.T ; R^{n+m+m \times d}\right) \times F_{N}^{2}\left(0, T ; R^{m}\right)$ which is stated in the following part.

$$
\begin{aligned}
& E \int_{0}^{T}\left|\widehat{x}^{\varepsilon}\right|^{2} d t \leq C_{1} E \int_{0}^{T}\left[\left|\widehat{y}^{\varepsilon}\right|^{2}+\left|\widehat{z}^{\varepsilon}\right|^{2}\right. \\
& \left.\quad+\int_{E}\left|\widehat{k}^{\varepsilon}(e)\right|^{2} \lambda(d e)\right] d t+C_{1} \delta^{2} E \int_{0}^{T}\left|\widehat{u}^{\varepsilon}\right|^{2} d t, \\
& E \int_{0}^{T}\left[\left|\widehat{y}^{\varepsilon}\right|^{2}+\left|\widehat{z}^{\varepsilon}\right|^{2}+\int_{E}\left|\widehat{k}^{\varepsilon}(e)\right|^{2} \lambda(d e)\right] d t \\
& \quad \leq C_{1} E \int_{0}^{T}\left|\widehat{x}^{\varepsilon}\right|^{2} d t+C_{1} \delta^{2} E \int_{0}^{T}\left|\widehat{u}^{\varepsilon}\right|^{2} d t,
\end{aligned}
$$

$$
\begin{aligned}
0 & =E \int_{0}^{T} d\left\langle G \widehat{x}^{\varepsilon}, \widehat{y}^{\varepsilon}\right\rangle=E \int_{0}^{T}\left\langle A\left(t, \mu_{\delta}, k_{\delta}^{\varepsilon}\right)\right. \\
& \left.-A\left(t, \widehat{\mu}_{\delta}, \widehat{k}_{\delta}^{\varepsilon}\right), \mu_{\delta}-\widehat{\mu}_{\delta}\right\rangle d t \\
& +E \int_{0}^{T} \int_{E}\left\langle G \widehat{g}, \widehat{k}^{\varepsilon}(e)\right\rangle \lambda(d e) d t+E \\
& \cdot \int_{0}^{T}\left[\left\langle G \widehat{b}_{\delta}, \widehat{y}^{\varepsilon}\right\rangle+\left\langle G \widehat{x}^{\varepsilon},-\widehat{f}_{\delta}\right\rangle+\left\langle G \widehat{\sigma}_{\delta}, \widehat{z}^{\varepsilon}\right\rangle\right. \\
& \left.+\int_{E}\left\langle G \widehat{g}_{\delta}, \widehat{k}^{\varepsilon}(e)\right\rangle \lambda(d e)\right] d t \\
& \leq-v_{1} E \int_{0}^{T}\left|G \widehat{x}^{\varepsilon}\right|^{2} d t-v_{2}\left[E \int_{0}^{T}\left|G^{\tau} \widehat{y}^{\varepsilon}\right|^{2}\right. \\
& \left.+\left|G^{\tau} \widehat{z}^{\varepsilon}\right|^{2}+\int_{E}\left|G^{\tau} \widehat{k}^{\varepsilon}(e)\right|^{2} \lambda(d e)\right] d t \\
& +C_{1} E \int_{0}^{T}\left[\left|\widehat{x}^{\varepsilon}\right|^{2}+\left|\widehat{y}^{\varepsilon}\right|^{2}+\left|\widehat{z}^{\varepsilon}\right|^{2}\right. \\
& \left.+\int_{E}\left|\widehat{k}^{\varepsilon}(e)\right|^{2} \lambda(d e)\right] d t+C_{2} E \int_{0}^{T} \delta^{2}\left|\widehat{u}^{\varepsilon}\right|^{2} d t .
\end{aligned}
$$


That is,

$$
\begin{aligned}
& v_{1} E \int_{0}^{T}\left|G \widehat{x}^{\varepsilon}\right|^{2} d t+v_{2} E \int_{0}^{T}\left[\left|G \widehat{y}^{\varepsilon}\right|^{2}+\left|G \widehat{z}^{\varepsilon}\right|^{2}\right. \\
& \left.+\int_{E}\left|G \widehat{k}^{\varepsilon}(e)\right|^{2} \lambda(d e)\right] d t \leq C_{1} E \int_{0}^{T}\left[\left|\widehat{x}^{\varepsilon}\right|^{2}\right. \\
& \left.+\left|\widehat{y}^{\varepsilon}\right|^{2}+\left|\widehat{z}^{\varepsilon}\right|^{2}+\int_{E}\left|\widehat{k}^{\varepsilon}(e)\right|^{2} \lambda(d e)\right] d t \\
& +C_{2} E \int_{0}^{T} \delta^{2}\left|\widehat{u}^{\varepsilon}\right|^{2} d t .
\end{aligned}
$$

If $m>n, v_{1}>0, v_{2} \geq 0$, we can get

$$
E \int_{0}^{T}\left|\widehat{x}^{\varepsilon}\right|^{2} d t \leq C_{3} \delta^{2} E \int_{0}^{T}\left|\widehat{u}^{\varepsilon}\right|^{2} d t .
$$

Let $\delta \rightarrow 0$; we can get $x_{\delta}^{\varepsilon} \rightarrow x^{\varepsilon}$, and from the unique solution of (6), we have $\left(y_{\delta}^{\varepsilon}, z_{\delta}^{\varepsilon}, k_{\delta}^{\varepsilon}\right)$ which converges to $\left(y^{\varepsilon}, z^{\varepsilon}, k^{\varepsilon}\right)$ in $\mathscr{M}^{2}\left(0, T ; R^{m+m \times d}\right) \times F_{N}^{2}\left(0, T ; R^{m}\right)$.

If $m<n, v_{1} \geq 0, v_{2}>0$, we can get

$$
\begin{gathered}
E \int_{0}^{T}\left[\left|\widehat{y}^{\varepsilon}\right|^{2}+\left|\widehat{z}^{\varepsilon}\right|^{2}+\int_{E}\left|\widehat{k}^{\varepsilon}(e)\right|^{2} \lambda(d e)\right] d t \\
\leq C_{4} \delta^{2} E \int_{0}^{T}\left|\widehat{u}^{\varepsilon}\right|^{2} d t .
\end{gathered}
$$

Let $\delta \rightarrow 0$; we also can get $\left(y_{\delta}^{\varepsilon}, z_{\delta}^{\varepsilon}, k_{\delta}^{\varepsilon}\right)$ which converges to $\left(y^{\varepsilon}, z^{\varepsilon}, k^{\varepsilon}\right)$ in $\mathscr{M}^{2}\left(0, T ; R^{m+m \times d}\right) \times F_{N}^{2}\left(0, T ; R^{m}\right)$, and then from the unique solution of (6), we have $x_{\delta}^{\varepsilon}$ which converge to $x^{\varepsilon}$.

Define $\Delta x^{\varepsilon}=\left(x_{\delta}^{\varepsilon}-x^{\varepsilon}\right) / \delta, \Delta y^{\varepsilon}=\left(y_{\delta}^{\varepsilon}-y^{\varepsilon}\right) / \delta, \Delta z^{\varepsilon}=$ $\left(z_{\delta}^{\varepsilon}-z^{\varepsilon}\right) / \delta$, and $\Delta k^{\varepsilon}=\left(k_{\delta}^{\varepsilon}-k^{\varepsilon}\right) / \delta$; then

$$
\begin{array}{r}
d \Delta x^{\varepsilon}=\frac{b\left(t, \bigwedge_{\delta}^{\varepsilon}, u^{\varepsilon}+\delta \widehat{u}^{\varepsilon}\right)-b\left(t, \bigwedge^{\varepsilon}, \widehat{u}^{\varepsilon}\right)}{\delta} d t \\
+\frac{\sigma\left(t, \bigwedge_{\delta}^{\varepsilon}, u^{\varepsilon}+\delta \widehat{u}^{\varepsilon}\right)-\sigma\left(t, \bigwedge^{\varepsilon}, \widehat{u}^{\varepsilon}\right)}{\delta} d B_{t} \\
+\frac{\int_{E}\left[g\left(t, \bigwedge_{\delta}^{\varepsilon}, u^{\varepsilon}+\delta \widehat{u}^{\varepsilon}\right)-g\left(t, \bigwedge^{\varepsilon}, \widehat{u}^{\varepsilon}\right)\right]}{\delta}
\end{array}
$$

$\widetilde{N}(d e, d t)$

$$
\begin{aligned}
& -d \Delta y^{\varepsilon}=\frac{f\left(t, \bigwedge_{\delta}^{\varepsilon}, u^{\varepsilon}+\delta \widehat{u}^{\varepsilon}\right)-f\left(t, \bigwedge^{\varepsilon}, \widehat{u}^{\varepsilon}\right)}{\delta} d t \\
& -\Delta z^{\varepsilon} d B_{t}+\frac{\int_{E} \widehat{k}_{\delta}^{\varepsilon}(e)}{\delta} \widetilde{N}(d e, d t),
\end{aligned}
$$

$$
\begin{aligned}
& \Delta x^{\varepsilon}(0)=0, \\
& \Delta y^{\varepsilon}(T)=0 .
\end{aligned}
$$

Sign

$$
\begin{aligned}
& K_{1}=\frac{L\left(t, \bigwedge_{\delta}^{\varepsilon}, u^{\varepsilon}+\delta \widehat{u}^{\varepsilon}\right)-L\left(t, x^{\varepsilon}, y_{\delta}^{\varepsilon}, z_{\delta}^{\varepsilon}, k_{\delta}^{\varepsilon}, u^{\varepsilon}+\delta \widehat{u}^{\varepsilon}\right)}{x_{\delta}^{\varepsilon}-x^{\varepsilon}}, \\
& K_{2} \\
& =\frac{L\left(t, x^{\varepsilon}, y_{\delta}^{\varepsilon}, z_{\delta}^{\varepsilon}, k_{\delta}^{\varepsilon}, u^{\varepsilon}+\delta \widehat{u}^{\varepsilon}\right)-L\left(t, x^{\varepsilon}, y^{\varepsilon}, z_{\delta}^{\varepsilon}, k_{\delta}^{\varepsilon}, u^{\varepsilon}+\delta \widehat{u}^{\varepsilon}\right)}{y_{\delta}^{\varepsilon}-y^{\varepsilon}}, \\
& K_{3} \\
& =\frac{L\left(t, x^{\varepsilon}, y^{\varepsilon}, z_{\delta}^{\varepsilon}, k_{\delta}^{\varepsilon}, u^{\varepsilon}+\delta \widehat{u}^{\varepsilon}\right)-L\left(t, x^{\varepsilon}, y^{\varepsilon}, z^{\varepsilon}, k_{\delta}^{\varepsilon}, u^{\varepsilon}+\delta \widehat{u}^{\varepsilon}\right)}{z_{\delta}^{\varepsilon}-z^{\varepsilon}}, \\
& K_{4}=\frac{L\left(t, \bigwedge_{\delta}^{\varepsilon}, u^{\varepsilon}+\delta \widehat{u}^{\varepsilon}\right)-L\left(t, x_{\delta}^{\varepsilon}, y_{\delta}^{\varepsilon}, z_{\delta}^{\varepsilon}, k^{\varepsilon}, u^{\varepsilon}+\delta \widehat{u}^{\varepsilon}\right)}{k_{\delta}^{\varepsilon}-k^{\varepsilon}}, \\
& K_{5}=\frac{L\left(t, \bigwedge_{\delta}^{\varepsilon}, u+\delta v\right)-L\left(t, \bigwedge_{\delta}^{\varepsilon}, u^{\varepsilon}\right)}{\delta \widehat{u}^{\varepsilon}},
\end{aligned}
$$

where $L$ denotes $b, \sigma$, and $f$, respectively, and

$$
\begin{aligned}
& K_{1}^{L}(t)= \begin{cases}K_{1}(t), & x_{\delta}^{\varepsilon}-x^{\varepsilon} \neq 0, \\
0, & \text { otherelse, }\end{cases} \\
& K_{2}^{L}(t)= \begin{cases}K_{2}(t), & y_{\delta}^{\varepsilon}-y^{\varepsilon} \neq 0, \\
0, & \text { otherelse, }\end{cases} \\
& K_{3}^{L}(t)= \begin{cases}K_{3}(t), & z_{\delta}^{\varepsilon}-z^{\varepsilon} \neq 0, \\
0, & \text { otherelse },\end{cases} \\
& K_{4}^{L}(t)= \begin{cases}K_{4}(t), & k_{\delta}^{\varepsilon}-k^{\varepsilon} \neq 0, \\
0, & \text { otherelse },\end{cases} \\
& K_{5}^{L}(t)= \begin{cases}K_{5}(t), & \delta \times v \neq 0, \\
0, & \text { otherelse }\end{cases}
\end{aligned}
$$

Let

$$
\begin{aligned}
\bar{L}(t, x, y, z, k, v)= & K_{1}(t) x+K_{2}(t) y+K_{3}(t) z \\
& +K_{4}(t) k+K_{5}(t) v,
\end{aligned}
$$

according to $\left(\mathrm{H}_{3}\right)$ and the result of continuity:

$$
\begin{aligned}
\lim _{\delta \rightarrow 0} \bar{L} & \left(t, \Delta x^{\varepsilon}, \Delta y^{\varepsilon}, \Delta z^{\varepsilon}, \Delta k^{\varepsilon}, \widehat{u}^{\varepsilon}\right) \\
& -L_{x}\left(t, x^{\varepsilon}, y^{\varepsilon}, z^{\varepsilon}, k^{\varepsilon}, u\right) \Delta x^{\varepsilon} \\
& -L_{y}\left(t, x^{\varepsilon}, y^{\varepsilon}, z^{\varepsilon}, k^{\varepsilon}, u\right) \Delta y^{\varepsilon} \\
& -L_{z}\left(t, x^{\varepsilon}, y^{\varepsilon}, z^{\varepsilon}, k^{\varepsilon}, u\right) \Delta z^{\varepsilon} \\
& -L_{k}\left(t, x^{\varepsilon}, y^{\varepsilon}, z^{\varepsilon}, k^{\varepsilon}, u\right) \Delta k^{\varepsilon} \\
& -L_{v}\left(t, x^{\varepsilon}, y^{\varepsilon}, z^{\varepsilon}, k^{\varepsilon}, u\right) \widehat{u}^{\varepsilon}=0 .
\end{aligned}
$$

From the unique solution of (20) and the continuity, we have $\left(\Delta x^{\varepsilon}, \Delta y^{\varepsilon}, \Delta z^{\varepsilon}, \Delta k^{\varepsilon}\right)$ which converges to $\left(x_{\delta}^{\prime}, y_{\delta}^{\prime}, z_{\delta}^{\prime}, k_{\delta}^{\prime}\right)$ in $\mathscr{M}^{2}\left(0, T ; R^{n+m+m \times d}\right) \times F_{N}^{2}\left(0, T ; R^{m}\right)$ as $\delta \rightarrow 0$. 
Proposition 7 (variational inequality). $u(\cdot)$ is the optimal control, and $(x(\cdot), y(\cdot), z(\cdot), k(\cdot, \cdot))$ are the trajectory of (6) corresponding to $u(\cdot)$. Assume condition $\left(\mathrm{H}_{3}\right)$ holds; then there exist $\theta_{T} \in R^{n_{1}}, \theta_{0} \in R^{m_{1}}$, and $\theta \in R$, which are not all for 0 , such that the following variational inequality holds:

$$
\begin{aligned}
\lim _{\delta \rightarrow 0} & \left\{\left\langle\theta_{T}, E\left[G_{1 x}(x(T)) x^{\prime}(T)\right]\right\rangle+\left\langle\theta_{0}, E\left[G_{0 y}(y(0)) y^{\prime}(0)\right]\right\rangle\right. \\
& +\theta\left\{E \int_{0}^{T} \int_{E}\left[l_{x}(t) x^{\prime}(t)+l_{y}(t) y^{\prime}(t)+l_{z}(t) z^{\prime}(t)+l_{k}(t) k^{\prime}(t, \cdot)+l\left(u_{\delta}^{\varepsilon}(t)\right)-l(u(t))\right] \lambda(d e) d t\right. \\
& \left.\left.+E\left[\Phi_{x}(x(T)) x^{\prime}(T)+\gamma_{y}(y(0)) y^{\prime}(0)\right]\right\}\right\} \geq 0,
\end{aligned}
$$

where $\left(x^{\prime}(t), y^{\prime}(t), z^{\prime}(t), k^{\prime}(t, \cdot)\right)$ are the solution of (20).

Proof. Based on (17), we can get

$$
\begin{aligned}
& F_{\varepsilon}^{2}\left(u_{\delta}^{\varepsilon}(t)\right)-F_{\varepsilon}^{2}\left(u_{\varepsilon}(t)\right)=\left[E\left|G_{1}\left(x^{u_{\delta}^{\varepsilon}}(T)\right)\right|^{2}+E\left|G_{0}\left(Y^{u_{\delta}^{\varepsilon}}(0)\right)\right|^{2}+E\left|J\left(u_{\delta}^{\varepsilon}(t)\right)-J(u(t))+\varepsilon\right|^{2}\right]-\left[E\left|G_{1}\left(x^{u^{\varepsilon}}(T)\right)\right|^{2}\right. \\
& \left.\quad+E\left|G_{0}\left(Y^{u^{\varepsilon}}(0)\right)\right|^{2}+E\left|J\left(u^{\varepsilon}(t)\right)-J(u(t))+\varepsilon\right|^{2}\right]=2 E\left\langle G_{1}\left(x^{u^{\varepsilon}}(T)\right), G_{1 x}\left(x^{u^{\varepsilon}}(T)\right) x_{\delta}^{\prime}\right\rangle+2 E\left\langle G_{0}\left(Y^{u^{\varepsilon}}(0)\right),\right. \\
& \left.\quad G_{0 y}\left(Y^{u^{\varepsilon}}(0)\right) y_{\delta}^{\prime}\right\rangle+2\left|J\left(u^{\varepsilon}(t)\right)-J(u(t))+\varepsilon\right| \\
& \quad \cdot\left\{E \int_{0}^{T} \int_{E}\left[l_{x}(t) x^{\prime}(t)+l_{y}(t) y^{\prime}(t)+l_{z}(t) z^{\prime}(t)+l_{k}(t) k^{\prime}(t, e)+l\left(u_{\delta}^{\varepsilon}(t)\right)-l(u(t))\right] \lambda(d e) d t+E\left[\Phi_{x}\left(x_{\delta}(T)\right)\right.\right. \\
& \left.\left.\quad \cdot x_{\delta}^{\prime}(T)\right]+\left[\gamma_{y}\left(y_{\delta}(0)\right) y_{\delta}^{\prime}(0)\right]\right\} .
\end{aligned}
$$

Due to $\lim _{\delta \rightarrow 0} F_{\varepsilon}\left(u_{\delta}^{\varepsilon}(t)\right)=F_{\varepsilon}\left(u^{\varepsilon}(t)\right)$, we have

$$
\begin{aligned}
& \lim _{\delta \rightarrow 0} \frac{F_{\varepsilon}\left(u_{\delta}^{\varepsilon}(t)\right)-F_{\varepsilon}\left(u^{\varepsilon}(t)\right)}{\delta}=\lim _{\delta \rightarrow 0} \frac{F_{\varepsilon}^{2}\left(u_{\delta}^{\varepsilon}(t)\right)-F_{\varepsilon}^{2}\left(u^{\varepsilon}(t)\right)}{\delta} \times \frac{1}{F_{\varepsilon}\left(u_{\delta}^{\varepsilon}(t)\right)+F_{\varepsilon}\left(u^{\varepsilon}(t)\right)}=\frac{1}{F_{\varepsilon}\left(u^{\varepsilon}(t)\right)} \lim _{\delta \rightarrow 0} E\left\langle G_{1}\left(x^{u^{\varepsilon}}(T)\right),\right. \\
& \left.G_{1 x}\left(x^{u^{\varepsilon}}(T)\right) x_{\delta}^{\prime}(t)\right\rangle+E\left\langle G_{0}\left(Y^{u^{\varepsilon}}(0)\right), G_{0 y}\left(Y^{u^{\varepsilon}}(0)\right) y_{\delta}^{\prime}(t)\right\rangle+\left|J\left(u^{\varepsilon}(t)\right)-J(u(t))+\varepsilon\right| \\
& \quad \cdot\left\{E \int_{0}^{T} \int_{E}\left[l_{x}(t) x^{\prime}(t)+l_{y}(t) y^{\prime}(t)+l_{z}(t) z^{\prime}(t)+l_{k}(t) k^{\prime}(t, e)+l\left(u_{\delta}^{\varepsilon}(t)\right)-l(u(t))\right] \lambda(d e) d t+E\left[\Phi_{x}\left(x_{\delta}(T)\right)\right.\right. \\
& \left.\left.\cdot x_{\delta}^{\prime}(T)\right]+\left[\gamma_{y}\left(y_{\delta}(0)\right) y_{\delta}^{\prime}(0)\right]\right\} .
\end{aligned}
$$

Let

$$
\begin{aligned}
\theta_{\varepsilon} & =\frac{1}{F_{\varepsilon}\left(u^{\varepsilon}(t)\right)}\left|J\left(u^{\varepsilon}(t)\right)-J(u(t))+\varepsilon\right|, \\
\theta_{\varepsilon}^{T} & =\frac{1}{F_{\varepsilon}\left(u^{\varepsilon}(t)\right)} E G_{1}\left(x^{u^{\varepsilon}}(T)\right),
\end{aligned}
$$$$
\theta_{\varepsilon}^{0}=\frac{1}{F_{\varepsilon}\left(u^{\varepsilon}(t)\right)} E G_{0}\left(Y^{u^{\varepsilon}}(0)\right)
$$

and according to Proposition 6, one can get 


$$
\begin{aligned}
& \lim _{\delta \rightarrow 0}\left\langle\theta_{\varepsilon}^{T}, E G_{1 x}\left(x^{u^{\varepsilon}}(T)\right) x_{\delta}^{\prime}(T)\right\rangle+\lim _{\delta \rightarrow 0}\left\langle\theta_{\varepsilon}^{0}, E G_{0 y}\left(y^{u^{\varepsilon}}(0)\right) y_{\delta}^{\prime}(0)\right\rangle \\
& \quad+\lim _{\delta \rightarrow 0} \theta_{\varepsilon}\left[\left\{E \int _ { 0 } ^ { T } \int _ { E } \left[l_{x}(t) x^{\prime}(t)+l_{y}(t) y^{\prime}(t)+l_{z}(t) z^{\prime}(t)+l_{k}(t) k^{\prime}(t, e)+\left(l\left(u_{\delta}^{\varepsilon}(t)\right)-l(u(t))\right] \lambda(d e) d t\right.\right.\right. \\
& \left.\left.\quad+E\left[\Phi_{x}\left(x_{\delta}(T)\right) x_{\delta}^{\prime}(T)\right]+\left[\gamma_{y}\left(y_{\delta}(0)\right) y_{\delta}^{\prime}(0)\right]\right\}\right] \geq \lim _{\delta \rightarrow 0} \frac{F_{\varepsilon}\left(u_{\delta}^{\varepsilon}(t)\right)-F_{\varepsilon}\left(u^{\varepsilon}(t)\right)}{\delta} \geq \lim _{\delta \rightarrow 0} \frac{-\sqrt{\varepsilon} d\left(u_{\delta}^{\varepsilon}(t), u^{\varepsilon}(t)\right)}{\delta} \\
& \quad=\lim _{\delta \rightarrow 0} \frac{-\sqrt{\varepsilon} E\left[\int_{0}^{T}\left|u_{\delta}^{\epsilon}(t)-u^{\varepsilon}(t)\right|^{2} d t\right]^{1 / 2}}{\delta} .
\end{aligned}
$$

From the definition of $F_{\varepsilon}(\cdot)$ and $\left|\theta_{\varepsilon}\right|^{2}+\left|\theta_{\varepsilon}^{T}\right|^{2}+\left|\theta_{\varepsilon}^{0}\right|^{2}=1$, we know that $\left(\theta_{\varepsilon}, \theta_{\varepsilon}^{T}, \theta_{\varepsilon}^{0}\right)$ has convergence subsequence whose limitation is $\left(\theta, \theta^{T}, \theta^{0}\right)$. Due to $\delta \rightarrow 0, u_{\delta}^{\varepsilon} \rightarrow u^{\varepsilon}$, it is easy to get $x_{\delta}^{\prime} \rightarrow x^{\prime}, y_{\delta}^{\prime} \rightarrow y^{\prime}, z_{\delta}^{\prime} \rightarrow z^{\prime}$, and $k_{\delta}^{\prime} \rightarrow$ $k^{\prime}$ in $\mathscr{M}^{2}\left(0, T, R^{n+m+m \times d}\right) \times F_{N}^{2}\left(0, T ; R^{m}\right)$. Let $\varepsilon \rightarrow 0$; then variational inequality (36) can be obtained.

\section{The Adjoint Equation and Maximum Principle}

In this paper, we consider the following adjoint FBSDE of system (6):

$$
\begin{aligned}
& d P(t)=\left[f_{y}^{\tau}\left(t, \prod, u(t)\right) P(t)-b_{y}^{\tau}\left(t, \prod, u(t)\right)\right. \\
& \text { - } Q(t)-\sigma_{y}^{\tau}\left(t, \prod, u(t)\right) R(t)-g_{y}^{\tau}\left(t, \prod, u(t)\right) \\
& \left.\cdot K(t, e)-l_{y}\left(t, \prod, u(t)\right)\right] d t \\
& +\left[f_{z}^{\tau}\left(t, \prod, u(t)\right) P(t)-b_{z}^{\tau}\left(t, \prod, u(t)\right) Q(t)\right. \\
& -\sigma_{z}^{\tau}\left(t, \prod, u(t)\right) R(t)-g_{z}^{\tau}\left(t, \prod, u(t)\right) K(t, e) \\
& \left.-l_{z}^{\tau}\left(t, \prod, u(t)\right)\right] d B_{t} \\
& +\int_{E}\left[-f_{k}^{\tau}\left(t, \prod, u(t)\right) P(t)\right. \\
& +b_{k}^{\tau}\left(t, \prod, u(t)\right) Q(t)+\sigma_{k}^{\tau}\left(t, \prod, u(t)\right) R(t) \\
& \left.+g_{k}^{\tau}\left(t, \prod, u(t)\right) K(t, e)-l_{k}\right] \widetilde{N}(d e, d t), \\
& -d Q(t)=\left[f_{x}^{\tau}\left(t, \prod, u(t)\right) P(t)\right. \\
& +b_{x}^{\tau}\left(t, \prod, k_{t}, u(t)\right) Q(t)+\sigma_{x}^{\tau}\left(t, \prod, u(t)\right) R(t) \\
& \left.+g_{x}^{\tau}\left(t, \prod, u(t)\right) K(t, e)+l_{x}\left(t, \prod, u(t)\right)\right] d t \\
& -R(t) d B_{t}-\int_{E} K(t, e) \widetilde{N}(d e, d t),
\end{aligned}
$$$$
0 \leq t \leq T
$$

$$
\begin{aligned}
& P(0)=-\gamma_{y}(y(0))-G_{0 y}^{\tau}(y(0)), \\
& Q(T)=\Phi_{x}(x(T))+G_{1 x}^{\tau}(x(T))-h_{x}^{\tau}(x(T)) P(T),
\end{aligned}
$$

where $\left(\prod\right)$ denote $(x(t), y(t), z(t), k(t, e))$.

From assumption $\left(\mathrm{H}_{3}\right)$, We can verify that variational equation (20) and adjoint equation (41) all satisfy assumptions $\left(H_{1}\right),\left(H_{2}\right)$. Thus it can be seen that there exists a unique adapted solution $(P(t), Q(t), R(t), K(t, e))$ in $\mathscr{M}^{2}\left(0, T ; R^{n+m+m \times d+m}\right) \times F_{N}^{2}\left(0, T ; R^{m}\right)$ satisfying (41).

Define the Hamiltonian function $H(\cdot)$ as follows:

$$
\begin{aligned}
& H(t, x, y, z, k(\cdot), v, P, Q, R, K) \\
&=\langle P(\cdot),-f(t, x, y, z, k(\cdot), v)\rangle \\
&+\langle Q(\cdot), b(t, x, y, z, k(\cdot), v)\rangle \\
&+\langle R(\cdot), \sigma(t, x, y, z, k(\cdot), v)\rangle \\
&+\int_{E}\langle K(\cdot, e), g(t, x, y, z, k(\cdot), v)\rangle \lambda(d e) \\
&+l(t, x, y, z, k(\cdot), v) .
\end{aligned}
$$

Denote $H(t) \equiv H(x(t), y(t), z(t), k(t, \cdot), P(t), Q(t), R(t)$, $K(t, \cdot))$, and we can rewrite (26) in the following Hamiltonian system's form:

$$
\begin{aligned}
d P(t)= & -H_{y}(t) d t-H_{z}(t) d B_{t} \\
& -\int_{E} H_{k}(t, e) \widetilde{N}(d e, d t), \\
-d Q(t)= & H_{x}(t) d t-R(t) d B_{t} \\
& -\int_{E} K(t, e) \widetilde{N}(d e, d t),
\end{aligned}
$$

$0 \leq t \leq T$

$$
\begin{aligned}
P(0)= & -\gamma_{y}(y(0))-G_{0 y}^{\tau}(y(0)) \\
Q(T)= & \Phi_{x}(x(T))+G_{1 x}^{\tau}(x(T)) \\
& -h_{x}^{\tau}(x(T)) P(T) .
\end{aligned}
$$


In the following part, we give the detailed proof about the necessary maximum principle which is given in [24].

Theorem 8 (necessary maximum principle; see [24]). Let $\left(H_{1}\right),\left(H_{2}\right)$, and $\left(H_{3}\right)$ hold, and $(x(\cdot), y(\cdot), z(\cdot), k(\cdot, \cdot), u(\cdot))$ is the solution to the optimal control; then one has

$$
\begin{aligned}
& \left\langle H_{v}(t, x(t), y(t), z(t), k(t), u(t), P(t), Q(t), R(t),\right. \\
& K(t, \cdot)), v(t)-u(t)\rangle d t \geq 0,
\end{aligned}
$$

$$
\forall v(\cdot) \in \mathscr{U}_{a d}, \text { a.e, a.s. }
$$

Proof. Apply Itô formula to $\left\langle Q(t), x^{\prime}(t)\right\rangle+\left\langle P(t), y^{\prime}(t)\right\rangle$, then one can get

$$
\begin{aligned}
& E \int_{0}^{T} d\left[\left\langle Q(t) x^{\prime}(t)\right\rangle+\left\langle P(t) y^{\prime}(t)\right\rangle\right]=E[\langle Q(T), \\
& \left.\quad x^{\prime}(T)\right\rangle+\left\langle P(T), y^{\prime}(T)\right\rangle-\left\langle Q(0), x^{\prime}(0)\right\rangle \\
& \left.\quad-\left\langle P(0), y^{\prime}(0)\right\rangle\right]=E \int_{0}^{T}\left\{\left[\left\langle P(t),-f_{v}(t) \widehat{u}^{\varepsilon}(t)\right\rangle\right.\right. \\
& \left.\quad+\left\langle Q(t), b_{v}(t) \widehat{u}^{\varepsilon}(t)\right\rangle+\left\langle R(t), \sigma_{v}(t) \widehat{u}^{\varepsilon}(t)\right\rangle\right] \\
& \quad+\left[-l_{x}(t) x^{\prime}(t)-l_{y}(t) y^{\prime}(t)-l_{z}(t) z^{\prime}(t)\right. \\
& \left.\left.\quad-l_{k}(t) k^{\prime}(t, \cdot)\right]\right\} d t ;
\end{aligned}
$$

that is,

$$
\begin{aligned}
E & {\left[\left\langle Q(T), x^{\prime}(T)\right\rangle+\left\langle P(T), y^{\prime}(T)\right\rangle-\langle Q(0),\right.} \\
& \left.\left.x^{\prime}(0)\right\rangle-\left\langle P(0), y^{\prime}(0)\right\rangle\right] \\
& =E \int_{0}^{T}\left\{\left[\left\langle P(t),-f_{v}(t) \widehat{u}^{\varepsilon}(t)\right\rangle\right.\right. \\
& +\left\langle Q(t), b_{v}(t) \widehat{u}^{\varepsilon}(t)\right\rangle+\left\langle R(t), \sigma_{v}(t) \widehat{u}^{\varepsilon}(t)\right\rangle \\
& \left.+\left\langle K(t, \cdot), g_{v}(t) \widehat{u}^{\varepsilon}(t)\right\rangle\right]+\left[-l_{x}(t) x^{\prime}(t)\right. \\
& \left.\left.-l_{y}(t) y^{\prime}(t)-l_{z}(t) z^{\prime}(t)-l_{k}(t) k^{\prime}(t, \cdot)\right]\right\} d t ;
\end{aligned}
$$

that is,

$$
\begin{aligned}
E & {\left[\left\langle Q(T), x^{\prime}(T)\right\rangle+\left\langle P(T), y^{\prime}(T)\right\rangle\right.} \\
& \left.-\left\langle Q(0), x^{\prime}(0)\right\rangle-\left\langle P(0), y^{\prime}(0)\right\rangle\right] \\
& +E \int_{0}^{T}\left[l_{x}(t) x^{\prime}(t)+l_{y}(t) y^{\prime}(t)+l_{z}(t) z^{\prime}(t)\right. \\
& \left.+l_{k}(t) k^{\prime}(t, \cdot)+l_{v}(t) \hat{u}^{\varepsilon}(t)\right] \\
& =E \int_{0}^{T}\left[\left\langle P(t),-f_{v}(t) \hat{u}^{\varepsilon}(t)\right\rangle\right.
\end{aligned}
$$

$$
\begin{aligned}
& +\left\langle Q(t), b_{v}(t) \widehat{u}^{\varepsilon}(t)\right\rangle+\left\langle R(t), \sigma_{v}(t) \widehat{u}^{\varepsilon}(t)\right\rangle \\
& \left.+\left\langle K(t, \cdot), g_{v}(t) \widehat{u}^{\varepsilon}(t)\right\rangle\right]+E \int_{0}^{T}\left\langle l_{v}(t), \widehat{u}^{\varepsilon}(t)\right\rangle d t \\
& =E \int_{0}^{T}\left\langle H_{v}(t), \widehat{u}^{\varepsilon}(t)\right\rangle d t .
\end{aligned}
$$

Due to

$$
\begin{aligned}
E & {\left[\left\langle Q(T), x^{\prime}(T)\right\rangle+\left\langle P(T), y^{\prime}(T)\right\rangle\right.} \\
& \left.-\left\langle Q(0), x^{\prime}(0)\right\rangle-\left\langle P(0), y^{\prime}(0)\right\rangle\right] \\
& +E \int_{0}^{T}\left[l_{x}(t) x^{\prime}(t)+l_{y}(t) y^{\prime}(t)+l_{z}(t) z^{\prime}(t)\right. \\
& \left.+l_{k}(t) k^{\prime}(t, \cdot)+l_{v}(t) \widehat{u}^{\varepsilon}(t)\right] d t \geq 0,
\end{aligned}
$$

we can get

$$
\begin{gathered}
E \int_{0}^{T}\left\langle H_{v}(t, x(t), y(t), z(t), k(t), u(t), P(t), Q(t), R(t),\right. \\
K(t)), v(t)-u(t)\rangle d t \geq 0,
\end{gathered}
$$

and then the following holds:

$$
\begin{aligned}
\left\langle H_{v}(t, x(t), y(t), z(t), k(t), u(t), P(t), Q(t), R(t),\right. & \\
K(t)), v(t)-u(t)\rangle d t \geq 0, & \forall v(\cdot) \in \mathscr{U}_{\mathrm{ad}} \text { a.e, a.s. }
\end{aligned}
$$

This finishes the proof of Theorem 8 .

Remark 9. In this paper, we discuss the global fully coupled FBSCS where the diffusion coefficients $\sigma$ and $g$ both contain the control variables $v(\cdot)$ and $k(t, \cdot)$. And under the constraint condition of [11], it is easy to check that if $k(t, \cdot)=0$ and $g(\cdot)=$ 0 , the result of [11] can be obtained.

\section{Example: Maximizing the Consumption Utility}

Consider two assets in the financial market. One of the assets is a risk-free asset; the other one is a risky asset (e.g., stock). The price processes are described by the following dynamics equations:

$$
\begin{aligned}
& d S_{0}(t)=S_{0}(t) r(t) d t, \\
& S_{0}(0)=1, \\
& d S(t) \\
& \quad=S_{t_{-}}\left[\mu(t) d t+\sigma(t) d B_{t}+\int_{E} \eta(t, e) \widetilde{N}(d e, d t)\right], \\
& \qquad \quad 0 \leq t \leq T,
\end{aligned}
$$

$S(0)=s>0$,

where we assume the following:

(i) The risk-free interest rate $r(t)$ is a nonnegative predictable and bounded deterministic scalar-valued process. 
(ii) $\mu(t), \sigma(t)$ are given $\mathscr{F}_{t}$-adapted process, where $\mu(t)>$ $r(t)$. And also $\eta(t, e)$ is bounded deterministic process. To ensure that $S(t)>0$ for all $t$, let $\eta(t, e)>-1$ for all $e \in E$ and $\int_{E} \eta^{2}(t, e) \nu(d e)<\propto$.

(iii) The investor can borrow or lend risk-free interest rate.

Assume that an investor whose initial wealth is $a>0$ decides to invest in stock with the amount of $\pi(t)$. Denote $x(t)$ and $y(t)$ by the investor's wealth process and consumption utility process, respectively. Suppose $c(t)$ is the consumption process and $c(t) \in \mathcal{U}_{\text {ad }}$; by (6) and (51), we have

$$
\begin{aligned}
d x(t)= & x(t)[(1-\pi(t)) r(t)+\pi(t) \mu(t)-c(t)] d t \\
& +x(t) \pi(t) \sigma(t) d B_{t} \\
& +\pi(t) \int_{E} \eta(t, e) \widetilde{N}(d e, d t), \\
x(0)= & a, \\
-d y(t)= & f(t, x(t), y(t), z(t), k(t, \cdot), c(t)) d t \\
& -z(t) d B_{t}-\int_{E} k(t, e) \widetilde{N}(d e, d t), \\
& 0 \leq t \leq T, y(T)=h(x(T)) .
\end{aligned}
$$

The investor's problem is to solve

$$
\begin{array}{cl}
\sup _{\tilde{c}} y^{\tilde{c}}(0), \\
\text { subject to } & E\left[\int_{0}^{T} \pi(t) \widetilde{c}(t) d t\right] \\
& \leq E\left[\int_{0}^{T} \pi(t) c(t) d t\right],
\end{array}
$$

(the budget constraint),

where $y(t)=y^{\widetilde{c}}(t)$ is the solution of the following BSDE:

$$
\begin{aligned}
&-d y(t)= f(t, x(t), y(t), z(t), k(t, \cdot), c(t)) d t \\
&-z(t) d B_{t}-\int_{E} k(t, e) \widetilde{N}(d e, d t), \\
& \quad 0 \leq t \leq T, \\
& y(T)=h(x(T))=0 .
\end{aligned}
$$

Let $h(x(T))=G_{1 x}(x(T))$; then the problem can be transformed into the following:

$$
\sup _{\tilde{c}} y^{\widetilde{c}}(0)
$$

with $E\left[G_{1 x}(x(T))\right]=0$ subject to $E\left[\int_{0}^{T} \pi(t) \widetilde{c}(t) d t\right] \leq$ $E\left[\int_{0}^{T} \pi(t) c(t) d t\right]$. Then this problem changes to be finding the optimal consumption process $c_{t}$ under the wealth terminal constraint and the budget constraint.
For $\varepsilon>0$ define the Lagrangian

$$
\mathscr{L}_{\varepsilon}(\widetilde{c})=y^{\widetilde{c}}(0)-\varepsilon E\left[\int_{0}^{T} \pi(t)(\widetilde{c(t)}-c(t)) d t\right] \text {. }
$$

Suppose we, for each $\varepsilon>0$, can find an optimal $\widetilde{c(t, \varepsilon)}$ such that

$$
\sup _{\widetilde{c}} \mathscr{L}_{\varepsilon}(\widetilde{c}(t))=\mathscr{L}_{\varepsilon}(\widetilde{c}(t, \varepsilon))
$$

with constraints. Also, suppose we can find $\widehat{\varepsilon}$ such that

$$
E\left[\int_{0}^{T} \pi(t)(\widetilde{c}(t, \widehat{\varepsilon})-c(t)) d t\right]=0
$$

Then

$$
\widetilde{c}^{*}:=\widetilde{c}(t, \widehat{\varepsilon})
$$

is optimal for the original constrained problem. To see this, note that for all $\widetilde{c}(t)$ we have

$$
\begin{aligned}
y^{\widetilde{c}(t, \widehat{\varepsilon})}(0)= & y^{\widetilde{c}(t, \widehat{\varepsilon})}(0) \\
& -\widehat{\varepsilon} E\left[\int_{0}^{T} \pi(t)(\widetilde{c}(t, \widehat{\varepsilon})-c(t)) d t\right] \\
= & \mathscr{L}_{\widehat{\varepsilon}}(\widetilde{c}(t, \widehat{\varepsilon})) \geq \mathscr{L}_{\widehat{\varepsilon}}(\widetilde{c}(t)) \\
= & y^{\widetilde{c}}(0)-\widehat{\varepsilon} E\left[\int_{0}^{T} \pi(t)(\widetilde{c(t)}-c(t)) d t\right] \\
\geq & y^{\widetilde{c}}(0) .
\end{aligned}
$$

In view of this, we can solve the original constrained problem (54) in the following steps.

Step 1. Maximize over all $\mathscr{L}(\widetilde{c})$ (without constraints), for each given $\varepsilon>0$. Call the maximum $\widetilde{c}(t, \varepsilon)$.

Step 2. Find $\widehat{\varepsilon}$ such that $E\left[\int_{0}^{T} \pi(t)(\widetilde{c}(t, \widehat{\varepsilon})-c(t))\right]=0$. Then $\widetilde{c}^{*}:=\widetilde{c}(t, \widehat{\varepsilon})$ can solve the original constrained problem.

We now apply this to problem (54). Thus we fix $\varepsilon$ and proceed to study the unconstrained problem (54) in the context of Section 4: consider the controlled FBSDE system consisting of

$$
\begin{aligned}
d x(t)= & 0, \\
x(0)= & a, \\
-d y(t)= & f(t, x(t), y(t), z(t), k(t, \cdot), c(t)) d t \\
& -z(t) d B_{t}-\int_{E} k(t, e) \widetilde{N}(d e, d t), \\
y(T)= & h(x(T))=0 .
\end{aligned}
$$


The performance function is

$$
J(\widetilde{c})=-\varepsilon E\left[\int_{0}^{T} \pi(t)^{2}(\widetilde{c}(t)-c(t)) d t\right]+y^{\widetilde{c}}(0),
$$

where $\varepsilon>0$ is the Lagrange multiplier. The Hamiltonian function about this problem is

$$
\begin{aligned}
& H(t, y(t), z(t), k(t, \cdot), \alpha(t), c(t)) \\
& =-\varepsilon \pi(t)^{2}(\widetilde{c}(t)-c(t)) \\
& \quad+\alpha(t) f(t, y(t), z(t), k(t, \cdot), c(t)),
\end{aligned}
$$

$$
\begin{aligned}
& \widehat{\alpha}(t)=\exp \left(\int_{0}^{t}\left\{\widehat{f}_{y}(s)-\frac{1}{2}\left(\widehat{f}_{z}(s)\right)^{2}+\int_{R_{0}}\left[\ln \left(1+\frac{d\left(\nabla_{k} \hat{f}\right)}{d v}(s, e)\right)-\frac{d\left(\nabla_{k} \widehat{f}\right)}{d v}(s, e)\right] \nu(d e)\right\} d s+\int_{0}^{t} \widehat{f}_{z}(s) d B_{s}\right. \\
& \left.\quad+\int_{0}^{t} \int_{E} \ln \left(1+\frac{d\left(\nabla_{k} \hat{f}\right)}{d v}(s, e)\right) \widetilde{N}(d s, d e)\right), \quad t \geq 0,
\end{aligned}
$$

where we use the simplified notation

$$
\widehat{f}_{y}(s)=f_{y}(s)(s, \widehat{y}(s), \widehat{z}(s), \widehat{k}(s, \cdot), \widehat{c}(s)), \text { etc, }
$$

and we assume that

$$
\frac{d\left(\nabla_{k} f\right)}{d v}(s, e)>-1 \quad \forall s, e, \text { a.s. }
$$

Maximizing the Hamiltonian with respect to $\tilde{c}$ gives the firstorder equation:

$$
-\varepsilon \pi(t)^{2}+\widehat{\alpha}(t) f_{\widetilde{c}}(t, \widehat{y}(t), \widehat{z}(t), \widehat{k}(t, \cdot), \widetilde{c}(t))=0
$$

Assume that for each $t, y$, and $z$, the function

$$
\tilde{c} \longrightarrow \frac{\partial f}{\partial c}(t, \tilde{c}, y, z, k)
$$

has an inverse, denoted by $(\partial f / \partial c)^{-1}(t, y, z, k)$. Then the solution $\widetilde{c}(t)$ of the first-order condition (75) can be written into

$$
\begin{aligned}
\widetilde{c} & =\widetilde{c}(t, \widehat{\alpha}(t), \widehat{y}(t), \widehat{z}(t), \widehat{k}(t, \cdot)) \\
& =\left(\frac{\partial f}{\partial \widetilde{c}}\right)^{-1}\left(t, \frac{\varepsilon \pi^{2}(t)}{\widehat{\alpha}(t)}, \widehat{y}(t), \widehat{z}(t), \widehat{k}(t, \cdot)\right)=: \widetilde{c}(t)
\end{aligned}
$$

and the adjoint equation is

$$
\begin{aligned}
& d \alpha(t)=\alpha(t)\left[f_{y}(t, x(t), y(t), z(t), k(t, \cdot), c(t)) d t\right. \\
& \quad+f_{z}(t, x(t), y(t), z(t), k(t, \cdot), c(t)) d B_{t} \\
& \quad+\int_{E} \frac{d\left(\nabla_{k} f\right)}{d v}(t, x(t), y(t), z(t), k(t, e), c(t))(e) \\
& \quad \cdot \widetilde{N}(d e, d t)], \quad 0 \leq t \leq T, \\
& \alpha(0)=1 .
\end{aligned}
$$

Let $\nabla_{k} f$ denote the Frechet derivative of $f$ with respect to $k$ and $\left(d\left(\nabla_{k} f\right) / d \nu\right)(\cdot, \cdot)(e)$ denote its Radon-Nikodym derivative with respect to $\nu$. If $\widehat{c}=\widehat{c}(t, \varepsilon)$ is optimal for a given Lagrange multiplier $\varepsilon$ with corresponding values $(\widehat{\alpha}(t), \widehat{y}(t)$, $\widehat{z}(t), \widehat{k}(t, \cdot))$, we get by the Itô form the solution of $(61)$; that is,
Substituting this into the adjoint equation for $\widehat{\alpha}(t)$, we get

$$
\begin{aligned}
& d \alpha(t)=\alpha(t)\left[\widehat{f}_{y}(t, \widetilde{c}(t)) d t+\widehat{f}_{z}(t, \tilde{c}(t)) d B_{t}\right. \\
& \left.\quad+\int_{E} \frac{d\left(\nabla_{k} f\right)}{d \nu}(t, \widetilde{c}(t))(e) \widetilde{N}(d e, d t)\right], \\
& \alpha(0)=1,
\end{aligned}
$$

where

$$
\begin{aligned}
& \widehat{f}(t, \widetilde{c}(t))=f_{y}(t, \widetilde{c}(\widehat{\alpha}(t), \widehat{y}(t), \widehat{z}(t), \widehat{k}(t, \cdot)), \widehat{y}(t), \\
& \widehat{z}(t), \widehat{k}(t, \cdot)), \text { etc. }
\end{aligned}
$$

This is coupled to the following BSDE for $(\hat{y}, \widehat{z}, \widehat{k})$ :

$$
\begin{aligned}
& -d \widehat{y}(t)=f(t, \tilde{c}(\widehat{\alpha}(t), \widehat{y}(t), \widehat{z}(t), \widehat{k}(t, \cdot)), \widehat{y}(t), \\
& \widehat{z}(t), \widehat{k}(t, \cdot)) d t-\widehat{z}(t) d B_{t}-\int_{E} \widehat{k}(t, e) \\
& \cdot \widetilde{N}(d e, d t), \quad 0 \leq t \leq T, \\
& \widehat{y}(T)=0 .
\end{aligned}
$$

According to Lemma $1,(\widehat{\alpha}(t), \widehat{y}(t), \widehat{z}(t), \widehat{k}(t, e))$ can be found as the solution of the fully coupled system of FBSDE (72) and (75). 
Remark 10. Assume the following.

(i) $f$ has the form

$$
\begin{aligned}
f(t, \tilde{c}, y, z, k)= & f_{0}(t, \widetilde{c}, y)-\frac{1}{2} A(y) z^{2} \\
& -\frac{1}{2} \int E_{0} A_{1}(y, e) k^{2}(e) v(d e),
\end{aligned}
$$

where $f_{0}$ does not depend on $z$ and $k$.

(ii) Wealth state constraints

$$
\begin{gathered}
d x(t)=0, \\
x(0)=0,
\end{gathered}
$$

and then the question of maximizing consumption utility has been solved in [20].

\section{Conclusion}

In this paper, the global fully coupled FBSCS driven by ItôLévy process is investigated, and the initial-terminal state needs to be restrained. By using the variational technique, we get the variational inequality. We also get the necessary condition of optimal solution about this stochastic control system. Finally, we use the result to discuss the problem of consumption recursive utility.

\section{Conflicts of Interest}

The authors declare that there are no conflicts of interest regarding the publication of this paper.

\section{Acknowledgments}

This work was supported in part by the National Natural Science Foundation of China (11271007, 61503222), in part by the SDUET Research Fund (2012kytd105), in part by Shandong Provincial Natural Science Foundation of China (ZR2016FM48), and in part by the Doctoral Fund of the Ministry of Education of China (20123718110010).

\section{References}

[1] A. Bensoussan, "Lectures on stochastic control," in Nonlinear Filtering and Stochastic Control, vol. 972 of Lecture Notes in Math, pp. 1-62, Springer, Heidelberg, Berlin, 1981.

[2] A. Bensoussan, "Stochastic maximum principle for distributed parameter systems," Journal of the Franklin Institute, vol. 315, pp. 384-406, 1983.

[3] S. Peng, "Backward stochastic differential equations and applications to optimal control," Applied Mathematics and Optimization, vol. 27, no. 2, pp. 125-144, 1993.

[4] S. G. Peng, "A general stochastic maximum principle for optimal control problems," SIAM Journal on Control and Optimization, vol. 28 , no. 4, pp. 966-979, 1990.
[5] J. Yong and X. Y. Zhou, Stochastic Controls: Hamiltonian Systems and HJB Equations, vol. 43 of Applications of Mathematics, Springer, New York, NY, USA, 1999.

[6] Z. Wu, "Maximum principle for optimal control problem of fully coupled forward-backward stochastic systems," Systems Science and Mathematical Sciences, vol. 11, no. 3, pp. 249-259, 1998.

[7] S. Peng and Z. Wu, "Fully coupled forward-backward stochastic differential equations and applications to optimal control," SIAM Journal on Control and Optimization, vol. 37, no. 3, pp. 825-843, 1999.

[8] S. Ji and X. Y. Zhou, "A maximum principle for stochastic optimal control with terminal state constraints, and its applications," Communications in Information and Systems, vol. 6, no. 4, pp. 321-338, 2006.

[9] X. Liu, Y. Li, and W. Zhang, "Stochastic linear quadratic optimal control with constraint for discrete-time systems," Applied Mathematics and Computation, vol. 228, pp. 264-270, 2014.

[10] Z. Wu and A. Xu, "The maximum principle for fully coupled forward backward stochastic control system with state constraints," Journal of Shandong University, vol. 35, no. 4, pp. 373-380, 2000.

[11] J. T. Shi, "The maximum principle for fully coupled forwardbackward stochastic control system under state contraints," Journal of Shandong University, vol. 42, no. 1, pp. 44-99, 2007.

[12] Q.X. Meng, "A maximum principle for optimal control problem of fully coupled forward-backward stochastic systems with partial information," Science in China Series A, vol. 52, no. 7, pp. $1579-1588,2009$.

[13] S. Ji and Q. M. Wei, "A maximum principle for fully coupled forward-backward stochastic control systems with terminal state constraints," Journal of Mathematical Analysis and Applications, vol. 407, pp. 200-210, 2013.

[14] D. Nualart and W. Schoutens, "Chaotic and predictable representations for Lévy processes with applications in finance," Stochastic Processes and their Applications, vol. 90, no. 1, pp.109122, 2000.

[15] D. Applebaum, Lévy Processes and Stochastic Calculus, Cambridge University Press, Cambridge, UK, 2004.

[16] B. Øksendal and A. Sulem, "Maximum principle for optimal control of forward-backward stochastic differential equations with jumps," SIAM Journal on Control and Optimization, vol. 48, no. 5, pp. 2945-2976, 2009.

[17] J. Shi and Z. Wu, "Maximum principle for forward-backward stochastic control system with random jumps and applications to finance," Journal of Systems Science and Complexity, vol. 23, no. 2, pp. 219-231, 2010.

[18] J. Shi, "Necessary conditions for optimal control of forwardbackward stochastic systems with random jumps," International Journal of Stochastic Analysis, vol. 2012, Article ID 258674, 50 pages, 2012.

[19] F. Zhang, M. N. Tang, and Q. X. Meng, "Stochastic maximum principle of forward-backward stochastic system driven by lévy process," Chinese Annals of Mathematics, vol. 35A, no. 1, pp. 83100, 2014.

[20] K. Aase, "Recursive Utility and Jump-Diffusions," NHH Dept. of Business and Management Science Discussion Paper, No. 2014/9, vol. 6, pp. 1-40, 2015.

[21] B. Øksendal and A. Sulem, "Risk minimization in financial markets modeled by Ito-Lévy processes," Afrika Matematika, vol. 26, no. 5, pp. 939-979, 2015. 
[22] Z. Wu, "Forward-backward stochastic differential equations with brownian motion and poisson process," Acta Mathematicae Applicatae Sinica Systems, vol. 15, no. 4, pp. 433-443, 1999.

[23] I. Ekeland, "On the variational principle," Journal of Mathematical Analysis and Applications, vol. 47, pp. 324-353, 1974.

[24] M. Liu and X. Wang, "Necessary conditions of forwardbackward stochastic control system with initial-terminal constraints," in Proceedings of the 35th Chinese Control Conference, (CCC '16), pp. 1668-1671, July 2016. 


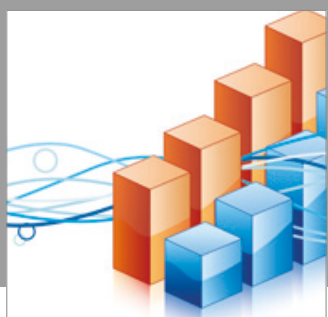

Advances in

Operations Research

vatersals

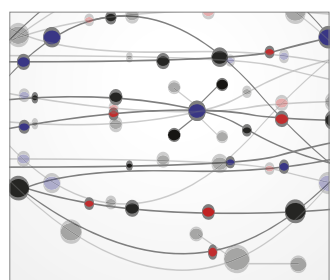

\section{The Scientific} World Journal
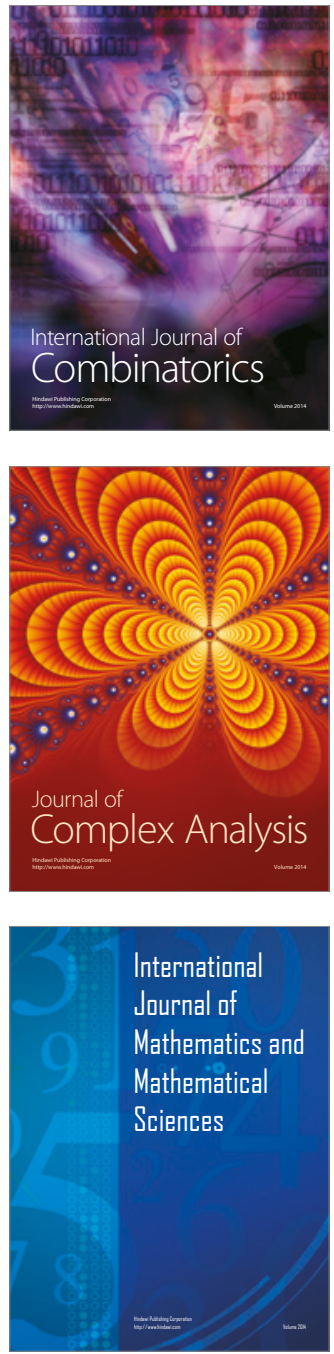
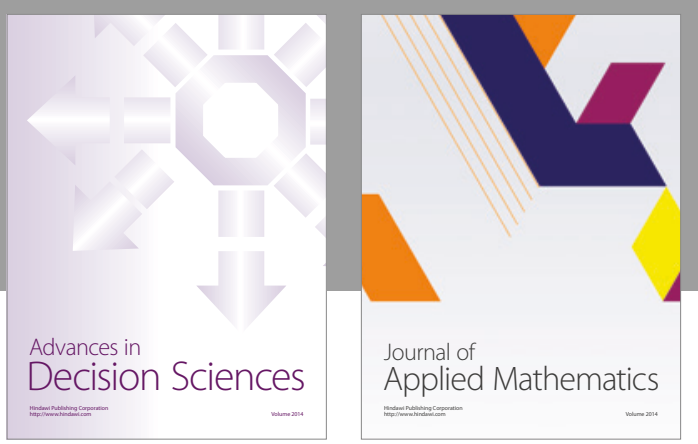

Algebra

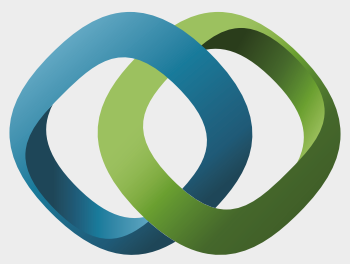

\section{Hindawi}

Submit your manuscripts at

https://www.hindawi.com
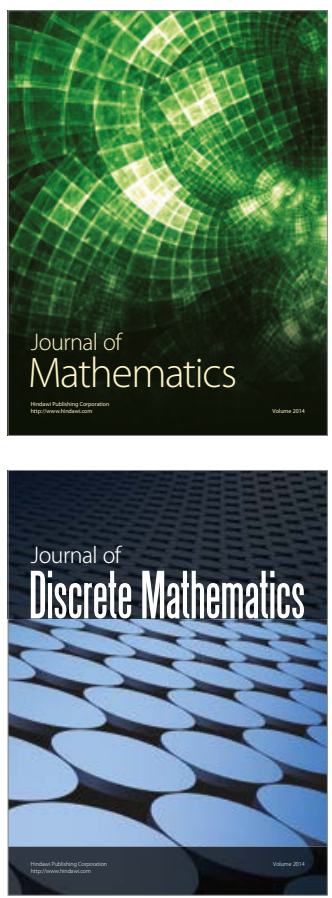

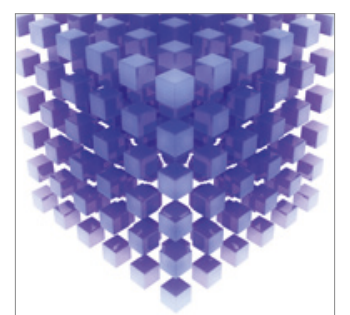

Mathematical Problems in Engineering
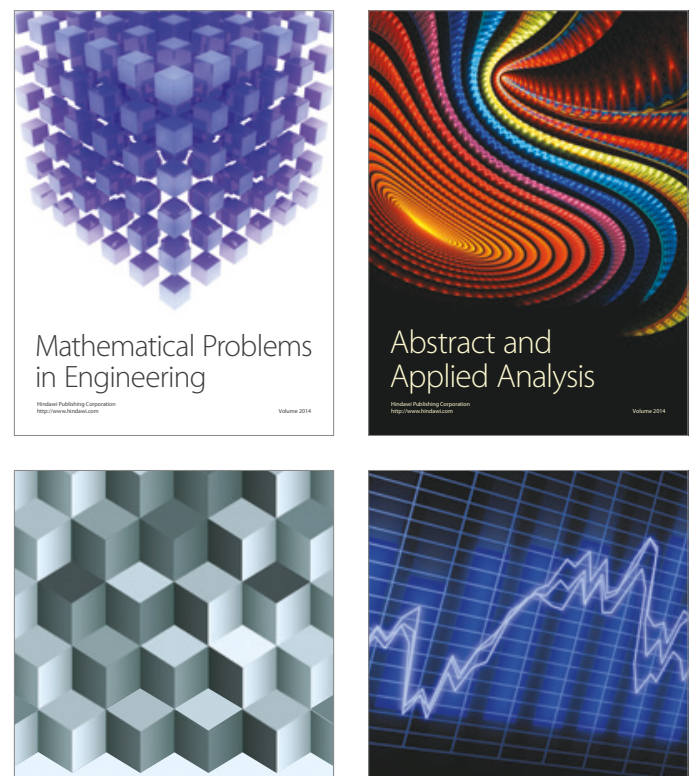

Journal of

Function Spaces

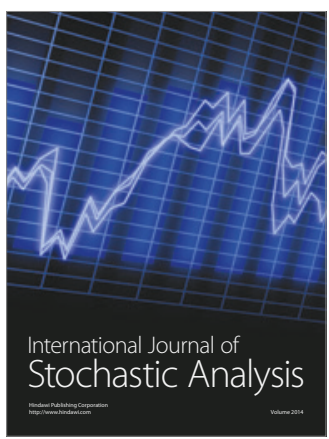

Probability and Statistics
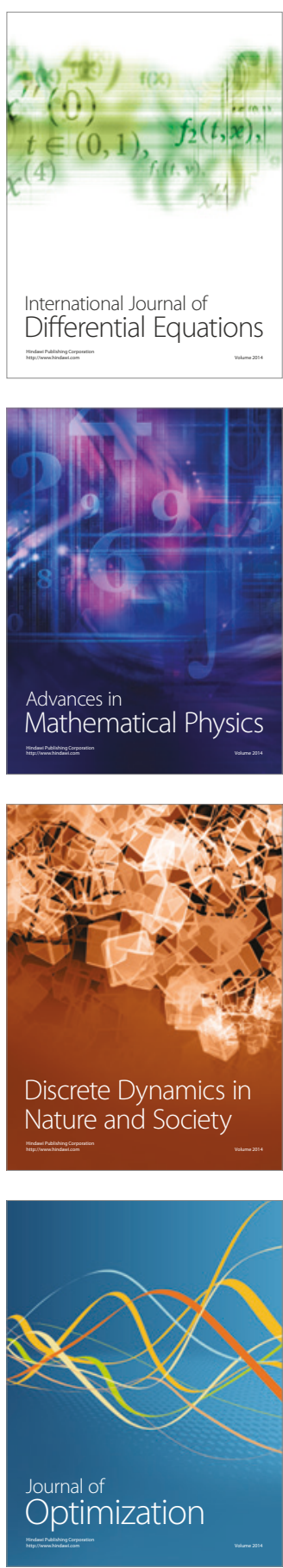